\title{
A Test of Learned Industriousness in the Physical Activity Domain
}

\author{
Eduardo E. Bustamante ${ }^{1}$, Catherine L. Davis ${ }^{1}, \&$ David X. Marquez ${ }^{2}$ \\ ${ }^{1}$ Georgia Prevention Institute, Medical College of Georgia, Georgia Regents University, Augusta, GA, USA \\ ${ }^{2}$ Department of Kinesiology \& Nutrition, University of Illinois at Chicago, Chicago, IL, USA \\ Correspondence: Eduardo E. Bustamante, Georgia Prevention Institute, Medical College of Georgia, Georgia \\ Regents University, 1120 15th Street, HS-1728, Augusta, Georgia 30912, USA. E-mail: ebustamante@gru.edu
}

Received: July 7, $2014 \quad$ Accepted: October 3, $2014 \quad$ Online Published: November 10, 2014

doi:10.5539/ijps.v6n4p12 URL: http://dx.doi.org/10.5539/ijps.v6n4p12

\begin{abstract}
Background: The Theory of Learned Industriousness states that durable individual differences in industriousness are due in part to differences in the extent to which individuals were rewarded for high effort at an earlier time. Individuals rewarded for high effort during training are thought to generalize greater persistence to subsequent tasks than those rewarded for low effort. This study tested whether rewarded physical and/or mental effort at different intensities generalized to greater persistence at a subsequent mental task. Methods: 80 inactive 18-25 year-olds were randomized into four groups: Low Mental Effort, High Mental Effort, Low Physical Effort, and High Physical Effort. Each completed group-specific effort training and a mental persistence task at baseline and posttest. Results: Factorial analysis of covariance revealed a significant domain $x$ effort interaction on persistence $(F[1,75]=4.93, p=.029)$. High Mental Effort and Low Mental Effort groups demonstrated similar gains in persistence $(d=-0.08, p>.05)$ and points earned $(d=0.11, p>.05)$ following effort training. High Physical Effort and Low Physical Effort diverged on persistence $(d=-0.49, p=.004)$ but not points earned $(d=-0.12, p>.05)$. Conclusions: Findings suggest either that training and test stimuli were too dissimilar to cue effects of associative learning in physical effort groups, or that effects were present but overpowered by the affective and neurocognitive consequences of an acute bout of intense aerobic physical activity. Findings do not support the Theory of Learned Industriousness nor generalization of effort across physical and mental domains.
\end{abstract}

Keywords: exercise, physical activity, learned industriousness, persistence

\section{Introduction}

The Theory of Learned Industriousness (Eisenberger, 1992) seeks to explain why some individuals consistently work harder than others of equal talent, ability, and motivation. This is important given that the best predictor of success at a given task is the effort and persistence put into it (Bandura, 1997). Indeed, industriousness and related constructs such as persistence, self-control, and self-discipline have implications for life trajectory, as research demonstrates them to be better predictors of social and academic functioning than standardized tests of intelligence or academic knowledge (Duckworth \& Seligman, 2005; Mischel, Shoda, \& Peake, 1988; Shoda, Mischel, \& Peake, 1990; Wolfe \& Johnson, 1995).

Eisenberger theorized that individual variations in industriousness, defined as the extent to which a person works hard or is productive at a given task, are due in part to differences in the extent to which individuals were rewarded for high effort in the past (Eisenberger, 1992). As a variant of operant conditioning, Learned Industriousness posits that the pairing of reinforcers with effortful exertion conditions the sensation of effort to take on secondary reward properties, thereby reducing the aversiveness of effort and leading to durable individual differences in industriousness across behaviors over the lifespan (Eisenberger, 1992).

A series of experiments examining associative learning in animals and humans provides the empirical foundation for the theory. Early investigations of associative learning documented a partial reinforcement extinction effect, in which animals rewarded for task performance on an intermittent schedule during training (i.e., reinforced for every $n^{\text {th }}$ task completed) had greater persistence at the task once the reinforcer was removed than those rewarded continuously (i.e., rewarded for each task completed) (Nation, Cooney, \& Gartrell, 1979). Later experiments found that individuals rewarded for high effort during training (i.e., completing difficult tasks in a single sitting or over a period of weeks) generalized greater persistence and productivity to subsequent tasks 
relative to those rewarded for lower effort (i.e., completing easier versions of the same tasks) (Boyagian, 1981; Eisenberger, Heerdt, Hamdi, Zimet, \& Bruckmeir, 1979; Eisenberger \& Leonard, 1980; Eisenberger, Mitchell, McDermitt, \& Masterson, 1984).

More recent applications of Learned Industriousness have focused on the use of monetary incentives to improve performance, interest, and creativity of students and workers (Cameron, Pierce, \& So, 2004; Eisenberger \& Aselage, 2008; Ventura, Shute, \& Zhao, 2013; Ventura \& Shute, 2013; Byron \& Khazanchi, 2012; Carter and McCullough, 2013); however, an intriguing aspect of the theory remains untested. Eisenberger (1992) hypothesized that both physical and mental exertion carry a similarly aversive sensation and a common energy reserve (James, 1890; McDougall, 1908), as evidenced by decrements in one following sufficient exhaustion of the other (Bonnet, 1980; Muraven, Tice, \& Baumeister, 1998). This implies that associative learning stemming from reinforcement of effort in either domain will generalize, to some degree, to the other domain through conditioning of the sensations associated with effortful exertion (Eisenberger, 1992).

If Learned Industriousness operates in both physical and mental domains then perhaps conditional reinforcement of high effort in physical activity (e.g., sport, dance, exercise, physical education, and martial arts programs) could be used to generalize high effort to other life domains (e.g., schoolwork). Longitudinal and cross-sectional data suggest that physical activity is associated with desirable behavioral outcomes, such as, improved self-regulation, academic achievement, on-task behavior, engagement in housework, and being employed outside of the home during summer (Oaten and Cheng, 2006; Coe, Pivarnik, Womack, Reeves, \& Malina, 2006; Hollar et al., 2010; Jarrett et al., 1998; Nelson \& Gordon-Larsen, 2006). However, the evidence is largely limited to observational studies and therefore the possibility that more industrious individuals are simply more likely to participate in physical activity cannot be discounted.

To date, the majority of studies investigating Learned Industriousness have focused on the influence of effort training on subsequent task persistence within the same domain, but not across domains. In other words, studies have examined either the influence of rewarded mental effort on mental persistence or rewarded physical effort on physical persistence, but not the influence of rewarded physical effort on subsequent mental persistence or vice versa. Only two studies have examined the influence of effort training on task persistence across domains and results from these studies are difficult to interpret due to equivocal findings and methodological limitations (Eisenberger et al., 1979; Boyagian \& Nation, 1981).

The first such study, Eisenberger (1979), rewarded depressed psychiatric patients for assisting staff with custodial responsibilities over a period of weeks. Participants praised for completing more difficult tasks demonstrated greater improvements in persistence from baseline at a card-sorting task than those praised for completing easier custodial tasks over the same time period (Eisenberger et al., 1979). Unfortunately, the results are difficult to interpret from a physical activity perspective because the extent to which the custodial tasks required physical versus mental effort was not clear.

The second, Boyagian and Nation (1981), utilized a 3-phase design in which participants underwent two brief effort trainings followed by a posttest extinction trial. A subset of participants completed two physical trainings followed by a physical extinction trial (human shuttle box), while a second subset completed one physical and one mental training followed by a mental extinction trial (unsolvable anagrams). The subset of participants tested on physical persistence during the extinction trial evidenced the expected effects of task difficulty, while those tested on anagram extinction did not (Boyagian, 1981). The authors concluded that the nondifferential results on anagram extinction were due to excessive dissimilarities between the training and test stimuli. However, these results are also difficult to interpret from a physical activity perspective because both mental and physical stimuli were utilized in effort training and both the training and test stimuli (button press and human shuttle box) require only minimal physical exertion.

This study utilized a randomized-controlled design to test whether participants rewarded for high effort at a single mental or physical task would generalize higher effort to a subsequent mental task than those rewarded for lower effort in the same activities. In accordance with Learned Industriousness, a main effect of effort was hypothesized, such that within each domain (i.e., physical and mental) groups rewarded for high effort during training would demonstrate greater persistence (primary outcome) and earn more rewards for good performance (secondary outcome) at the subsequent mental task than those rewarded for low effort (i.e., High Physical Effort > Low Physical Effort, High Mental Effort > Low Mental Effort). It was further hypothesized that a main effect of domain would also arise, such that groups rewarded for mental effort during training would perform better on a mental task after training than those rewarded for physical effort (i.e., High Mental Effort $>$ High Physical Effort, Low Mental Effort > Low Physical Effort) due to training specificity (Eisenberger, 1992). 
Finally, it was hypothesized that the anticipated advantage of high effort over low effort training would not vary by domain, nor would the anticipated advantage of mental over physical training vary by intensity; in other words, there would be no interaction of domain and effort.

\section{Methods}

\subsection{Study Design}

The study design was an adaptation of Eisenberger and Leonard (1980, Experiment 1), a lab-based study that randomized participants into four groups: Simple, Complex, Unsolvable, and Control (no effort training). Each treatment group received a unique effort training in which they were presented with a set of anagrams of varying degrees of difficulty, and rewarded with a point on a scoreboard accompanied by an audible beep for each solved correctly. Effort training was followed by a cartoon-comparison task during which participants were discreetly timed. The experiment was one of the first to demonstrate that the degree of cognitive effort required per reinforcer during mental effort training affected subsequent persistence at a different mental task.

We utilized two conditions from the original experiment (Simple and Complex) and re-labeled them Low Mental Effort and High Mental Effort. The current study also added two conditions that were not included in Eisenberger and Leonard (1980, Experiment 1): Low Physical Effort (a light-intensity treadmill bout) and High Physical Effort (a vigorous intensity treadmill bout). These physical effort groups were designed to emulate the mental effort groups in that they required different degrees of effort per reinforcer, and matched their maximum duration (10-min.) and achievable reinforcers (ten) during training. Therefore, the current study contained four groups: Low Mental Effort, High Mental Effort, Low Physical Effort, and High Physical Effort. These four groups constitute a $2 \times 2$ factorial design: two levels for effort (high and low) and two levels for domain (physical and mental). The Eisenberger and Leonard (1980, Experiment 1)protocols were utilized with the exception that a baseline measure of persistence was added to strengthen internal validity and statistical power, and a rest period was added between activities to allow heart rate in the High Physical Effort group to recover prior to posttest. Each group completed the same baseline persistence task (cartoon-comparison task) followed by group-specific effort training (anagrams or treadmill), and then repeated the persistence task (cartoon-comparison task) at posttest (see Figure 1). 


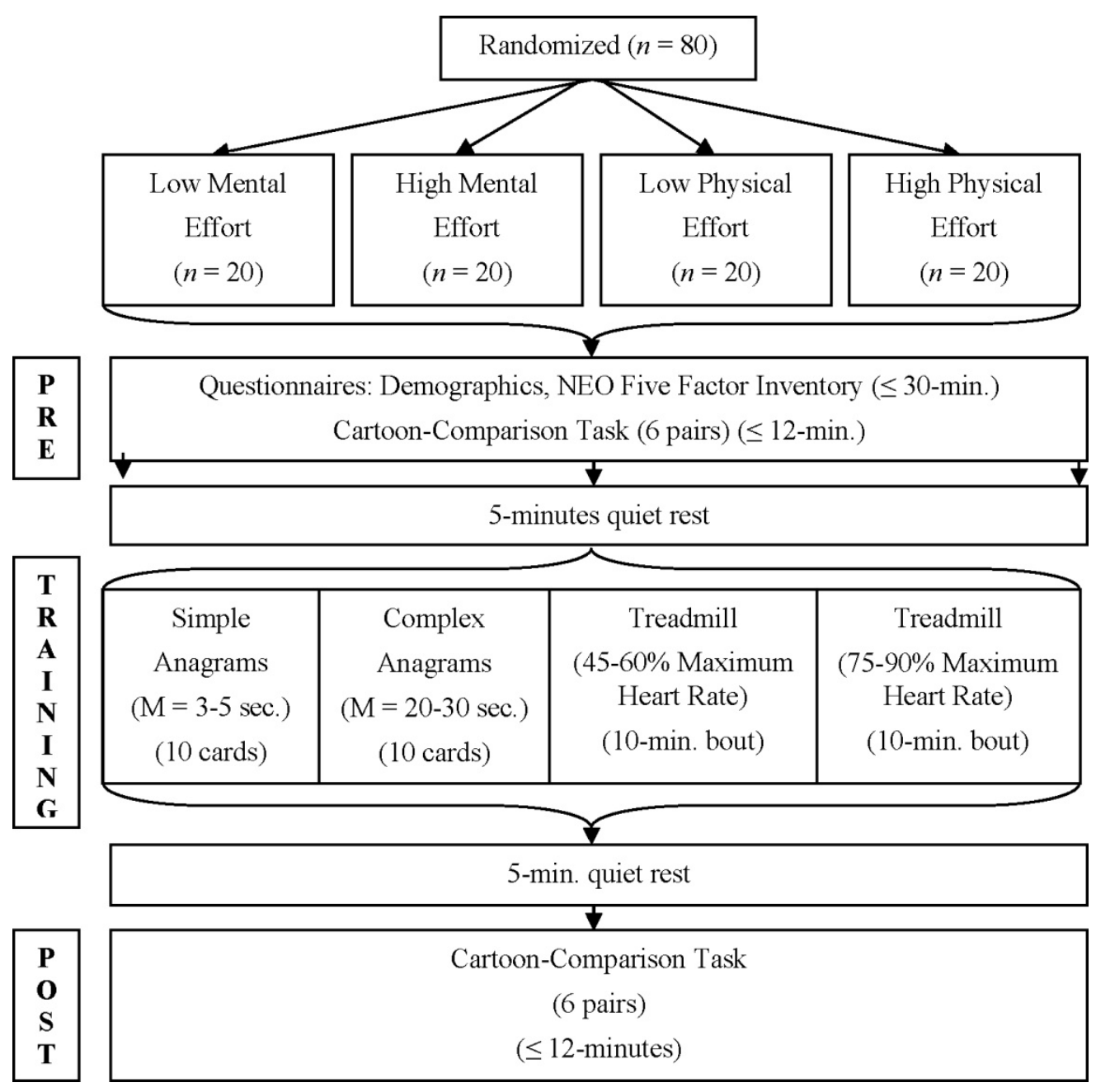

Figure 1. Experimental design

\subsection{Participants}

Participants included 80 physically inactive 18-25 year-olds who met American College of Sports Medicine criteria for low risk to participate in PA (Thayer, 2001). Participants were recruited by e-mail, flyers, internet announcements, word of mouth, lecture hall presentations, student club presentations, and the University's Department of Psychology subject pool. Inclusion criteria included self-reports of: 1) Age 18 to 25;2) participate in $\leq 3$ bouts of moderate-vigorous PA/week; 3 ) absence of obesity, hypertension, hypercholesterolemia, cigarette smoking, hypercholesterolemia, impaired fasting glucose, and family history of heart disease.

\subsection{Procedures}

All study activities were approved by the University of Illinois at Chicago Institutional Review Board. Following eligibility screening, random allocation software (http://www.randomizer.org) was used to assign 20 participants to each of 4 groups. Each participant was then scheduled for a testing session in a quiet room with the experimenter and one additional research staff member. Participants were asked to bring appropriate footwear and abstain from caffeine and exercise on the day of testing. Upon arrival, participants completed an Informed Consent document and two questionnaires: 1) Demographics, including self-reports of age (years), race, ethnicity, gender, education (highest level attained), marital status, body mass index (BMI), American College Test (ACT) score, and undergraduate grade point average (GPA). Consent and questionnaires took approximately 20-30 minutes to complete. Upon completion, participants were fitted with a Polar T31-Coded Heart Rate Monitor chest strap and engaged in a 5-min. quiet rest before beginning the persistence task. 


\subsubsection{Mental Persistence Task}

The mental persistence task was administered at baseline and posttest. Pairs of cartoon pictures that each differed in six details were used across groups. Twelve cartoon pairs were drawn randomly from two compilation books of cartoon-comparison tasks published in major newspapers under the title "Hocus Focus" (Kaufman \& Schroeter, 1982a, 1982b). Detailed procedures and task instructions are presented in the Appendix. Half of the pairs (six) were presented to participants at baseline and the other half (six) at posttest. The order of presentation of sets was counterbalanced evenly within each group. A manual scoreboard was visible to participants and used to signal correct responses. The experimenter sat at the opposite end of a table from study participants, read task instructions, and controlled the scoreboard, while a second research staff member sat behind the participants and recorded responses, heart rate, and discreetly timed the participants using a muted Polar E-600 heart rate monitor. The primary outcome, persistence, was operationalized as the total number of seconds between the participant turning over the first card to placing the last card face down.

During the mental persistence task, participants were allowed up to 120 -seconds and could earn up to six points on each card by identifying all six differences between the pairs of drawings. If a participant spent longer than 120 -seconds on a card, he or she was told, "Please go on to the next card." Participants were not aware that they were being timed and were not informed of the number of differences on each card. If participants asked they were told that there was no time limit and unlimited differences between cards. Given the absence of time and difference limits, the most adaptive response by participants to attain as many rewards as possible was to keep looking for differences without quitting. Upon completion of the baseline mental persistence measure participants engaged in 5-minutes of quiet rest, after which each group participated in a group-specific effort training intervention. After completion of the effort training intervention, each group engaged in a second 5-minute quiet rest to allow heart rate in the High Physical Effort group to decrease before repeating the mental persistence task at posttest.

\subsubsection{Effort Training}

During effort training, the Low Mental Effort and High Mental Effort groups were each presented with a set of ten 5 x 8-inch index cards, each containing a different printed five-letter anagram. Anagrams in the Low Mental Effort set had median solution times ranging from 3-5 seconds, whereas those in the High Mental Effort set had median solution times of 20-30 seconds (Tresselt \& Mayzner, 1966). As in the mental persistence task, the experimenter sat at the opposite end of the table from the participants, read task instructions, and controlled the scoreboard, while the second research staff member sat behind the participants and recorded responses, monitored heart rate, and discreetly timed the participants using the muted heart rate monitor. Similarly, as in the persistence measure, participants were not aware that they were being timed. During effort training a correct response was accompanied by brief non-specific verbal praise/encouragement (e.g., "good job") in addition to a point on the scoreboard for each anagram solved correctly. If a participant spent longer than 60 -seconds on a card, he or she was told to, "Please go on to the next card."

During effort training, High Physical Effort and Low Physical Effort participants were asked to walk, jog, or run on a treadmill at a predetermined heart rate range for 10-minutes. Intensity was determined by using the percentage of age-determined (220-participant age) maximum heart rate (Fox, Naughton, \& Haskell, 1971). The Low Physical Effort group was asked to keep their heart rate between 45 and $60 \%$ of their maximum heart rate coinciding with a Borg Rating of Perceived Exertion scale approximating "Very Light" to "Somewhat Hard" (9-12), while High Physical Effort participants were asked to keep their heart rate between 75 and $90 \%$ of their maximum coinciding with a Borg Rating of Perceived Exertion scale approximating "Hard" to "Extremely Hard" (15-18). Participants in both groups received a point on the scoreboard and brief verbal praise/encouragement for each minute that they successfully remained on the treadmill at the desired intensity. Heart Rate was monitored by a research staff member who held the heart rate monitor and informed the participants of their heart rate after each minute. Participants were asked to self-report their perceived exertion during the 5th and 9th minutes using the Borg Rating of Perceived Exertion scale.

\subsection{Statistical Analyses}

Statistical analyses were conducted with the Statistical Package for the Social Sciences, version 21. Descriptive analyses included means and standard deviations of participant characteristics. One-way analysis of variance (ANOVA) tested group differences in continuous baseline characteristics (i.e., age, BMI, undergraduate GPA, ACT score, and education), effort training duration, heart rate, and points earned, while $\chi^{2}$ tests assessed difference between groups on nominal (i.e., gender, race, and ethnicity) and ordinal (i.e., education) baseline characteristics. Both baseline and posttest persistence were right skewed (baseline skewness $=1.02$, standard 
error $=0.27$; posttest skewness $=0.75$, standard error $=0.27$ ). Boxplots of unadjusted baseline and posttest persistence means revealed four baseline and four posttest outliers, as evidenced by a Cook's distance $>.05$ and Leverage $>.075$. Therefore, a logarithmic transformation was used to normalize distributions on both baseline and posttest persistence (post-transformation baseline skewness $=.20$, standard error $=.27$; posttest skewness $=$ -.01 , standard error $=.27$, with no outliers).

Intent-to-treat factorial analysis of covariance (ANCOVA) tested a priori hypotheses of group differences by domain (physical effort groups vs. mental effort groups), effort (high effort groups vs. low effort groups), and their interaction, domain x effort, on the primary outcome, posttest persistence, adjusting for baseline persistence. The factorial ANCOVA was not run on the secondary outcome, points earned, because baseline and posttest points earned were not related $(r=-.06, p=.61)$. Statistical significance was assessed at $p<.05$. Additional participant characteristics were considered as covariates based upon whether they were related to the outcome and significantly different between groups; none met both criteria. As a sensitivity analysis, a ranked factorial ANCOVA (dependent variable: posttest persistence [rank-transformed]; covariate: baseline persistence [rank-transformed]; factors: domain, effort, \& domain x effort) was conducted (Conover \& Iman, 1982). Withinand between-group effect sizes, Cohen's $d$ (Cohen, 1988), were calculated on both persistence and points earned using unadjusted data. A positive sign was assigned to within-group effect sizes reflecting adaptive change and between-group effect sizes in the hypothesized direction.

\section{Results}

Participants were primarily female (70\%) undergraduate students $(65 \%)$ with normal BMI $(M=23.1, S D=3.3)$ and good GPA $(M=3.2, S D=.4)($ see Table 1$)$.

Table 1. Participant characteristics, \% or M (SD), by group

\begin{tabular}{|c|c|c|c|c|c|}
\hline $\begin{array}{l}\text { Participant } \\
\text { Characteristics }\end{array}$ & $\begin{array}{l}\text { Low Mental } \\
\text { Effort } \\
(n=20)\end{array}$ & $\begin{array}{l}\text { High Mental } \\
\text { Effort } \\
(n=20)\end{array}$ & $\begin{array}{l}\text { Low Physic } \\
\text { Effort } \\
(n=20)\end{array}$ & & $\begin{array}{l}\text { High Physical } \\
\text { Effort } \\
(n=20)\end{array}$ \\
\hline$\%$ Female & 75 & 70 & 65 & & 70 \\
\hline Age (yrs) & $21.2(2.3)$ & $21.3(2.1)$ & $20.6(2.4)$ & & $20.3(1.8)$ \\
\hline Body Mass Index (BMI) $\left(\mathrm{kg} / \mathrm{m}^{2}\right)$ & $22.5(7.4)$ & $24.3(3.3)$ & $22.5(2.8)$ & & $23.3(2.9)$ \\
\hline Undergraduate Grade Point Average & $3.3(0.3)$ & $3.1(0.3)$ & $3.2(0.5)$ & & $3.0(0.5)$ \\
\hline $\begin{array}{l}\text { American College Test (ACT) } \\
\text { Score }\end{array}$ & $26.0(4.0)$ & $22.3(2.8)$ & $24.6(3.9)$ & & $23.7(3.5)$ \\
\hline \multicolumn{6}{|l|}{ Ethnicity } \\
\hline$\%$ Latino/a & 25 & 50 & 35 & 55 & \\
\hline \multicolumn{6}{|l|}{ Race } \\
\hline$\%$ Native American & 0 & 0 & 5 & 0 & \\
\hline$\%$ Asian/Pacific-Islander & 30 & 15 & 20 & 20 & \\
\hline$\%$ African-American & 5 & 20 & 5 & 5 & \\
\hline$\%$ White & 50 & 30 & 45 & 25 & \\
\hline$\%$ Not Responding & 15 & 35 & 25 & 50 & \\
\hline \multicolumn{6}{|l|}{ Education (Highest Level Attained) } \\
\hline \% High School Graduate & 10 & 0 & 20 & 10 & \\
\hline$\% 1-3$ years college & 50 & 65 & 60 & 85 & \\
\hline$\%$ College/Univ. Graduate & 40 & 35 & 20 & 5 & \\
\hline
\end{tabular}

There were no significant differences between groups on participant characteristics $(p \mathrm{~s}>.05)$. Table 2 presents means and standard deviations for variables measured during effort training. All participants in physical effort groups successfully completed the 10 -minutes $(600-\mathrm{sec}$.) on the treadmill without exception. A One-way 
ANOVA revealed significant differences between groups in effort training duration $(\mathrm{F}[3,76]=204.9, p<.05)$, points earned $(\mathrm{F}[3,76]=30.4, p<.05)$, effort training $\mathrm{HR}(\mathrm{F}[3,75]=322.1, p<.05)$, and posttest $\mathrm{HR}(\mathrm{F}[3,75]$ $=16.6, p<.05)$. An independent samples $t$-test revealed a significant difference favoring High Physical Effort over Low Physical Effort on average RPE score during effort training $(t=-4.7, d f=34.7, p<.05)$.

Table 2. Duration, points earned, heart rate, and rating of perceived exertion during training, and posttest heart rate by group, $\mathrm{M}(\mathrm{SD})$

\begin{tabular}{lllll}
\hline & $\begin{array}{l}\text { Low Mental } \\
\text { Effort } \\
(n=20)\end{array}$ & $\begin{array}{l}\text { High Mental } \\
\text { Effort } \\
(n=20)\end{array}$ & $\begin{array}{l}\text { Low Physical } \\
\text { Effort } \\
(n=20)\end{array}$ & $\begin{array}{l}\text { High Physical } \\
\text { Effort } \\
(n=20)\end{array}$ \\
\hline Duration (seconds) & $135.1(99.9)$ & $238.9(37.7)$ & $600.0(0.0)^{a}$ & $600.0(0.0)^{a}$ \\
Points Earned & $8.1(1.5)$ & $6.0(2.2)$ & $9.7(1.0)^{b}$ & $9.9(0.4)^{b}$ \\
Effort Training Heart Rate & $81.2(2.8)^{c}$ & $81.4(11.8)^{c}$ & $106.8(7.6)$ & $165.2(6.8)$ \\
Posttest Heart Rate (beats per min.) & $80.0(10.7)^{d}$ & $81.0(11.6)^{d}$ & $78.8(8.2)^{d}$ & $98.6(10.4)$ \\
Borg Rating of Perceived Exertion & $\mathrm{NA}$ & NA & $9.4(2.0)$ & $12.1(1.5)$
\end{tabular}

Note. Values sharing a superscript letter are not significantly different from one another at critical value $p<.05$.

Table 3 presents unadjusted means, standard deviations, and within-group effect sizes for persistence and points earned across groups at baseline and posttest. There was a moderate between-group effect size favoring Low Physical Effort over High Physical Effort on persistence $(d=-.49)$, while other between-group effect sizes on persistence were either small, $d=-0.22$ (Low Physical Effort $>$ Low Mental Effort) and $d=0.19$ (High Mental Effort $>$ High Physical Effort), or near zero, $d=-0.08$ (Low Mental Effort > High Mental Effort).

Table 3. Unadjusted means and standard deviations and within-group effect sizes (Cohen's d) for persistence and points earned

\begin{tabular}{|c|c|c|c|c|c|}
\hline & & $\begin{array}{l}\text { Low Mental } \\
\text { Effort } \\
(n=20)\end{array}$ & $\begin{array}{l}\text { High Mental } \\
\text { Effort } \\
(n=20)\end{array}$ & $\begin{array}{l}\text { Low Physical } \\
\text { Effort } \\
(n=20)\end{array}$ & $\begin{array}{l}\text { High Physical } \\
\text { Effort } \\
(n=20)\end{array}$ \\
\hline \multicolumn{6}{|l|}{ Persistence } \\
\hline Baseline & & $253.3(98.8)$ & $298.3(141.3)$ & 304.7 (104.9) & $283.0(121.4)$ \\
\hline Posttest & & $297.2(149.1)$ & $331.1(138.6)$ & $375.4(127.7)$ & $289.2(167.3)$ \\
\hline Size $^{\text {Within-Group }}$ & Effect & 0.35 & 0.23 & 0.61 & 0.04 \\
\hline \multicolumn{6}{|l|}{ Points Earned } \\
\hline Baseline & & $18.2(6.2)$ & $18.8(7.8)$ & $19.1(4.6)$ & $18.3(6.0)$ \\
\hline Posttest & & $20.4(6.3)$ & $21.7(4.9)$ & $21.8(7.3)$ & $20.3(5.8)$ \\
\hline Size $^{\text {Within-Group }}$ & Effect & 0.35 & 0.46 & 0.45 & 0.34 \\
\hline
\end{tabular}

Figure 2 presents baseline means and standard errors and adjusted posttest geometric means and standard errors (Bland \& Altman, 1996) for persistence across groups. The parametric factorial ANCOVA revealed a main effect of effort ( $\mathrm{F}[1,75]=4.04, p=.048)$, but not domain ( $\mathrm{F}[1,75]=0.05, p=.591)$, and a significant interaction for domain $x$ effort $(\mathrm{F}[1,75]=4.93, p=.029)$. Investigation of simple effects revealed that Low Physical Effort outperformed High Physical Effort $(p=.004)$, while the other groups did not differ $(p \mathrm{~s}>.05)$. Similar results were obtained with the nonparametric analysis (effort: $\mathrm{F}[1,75]=4.88, p=.03$; domain: $\mathrm{F}[1,75]$ $=0.48, p=.49)$, domain $x$ effort: $\mathrm{F}[1,75]=6.11, p=.016)$. 

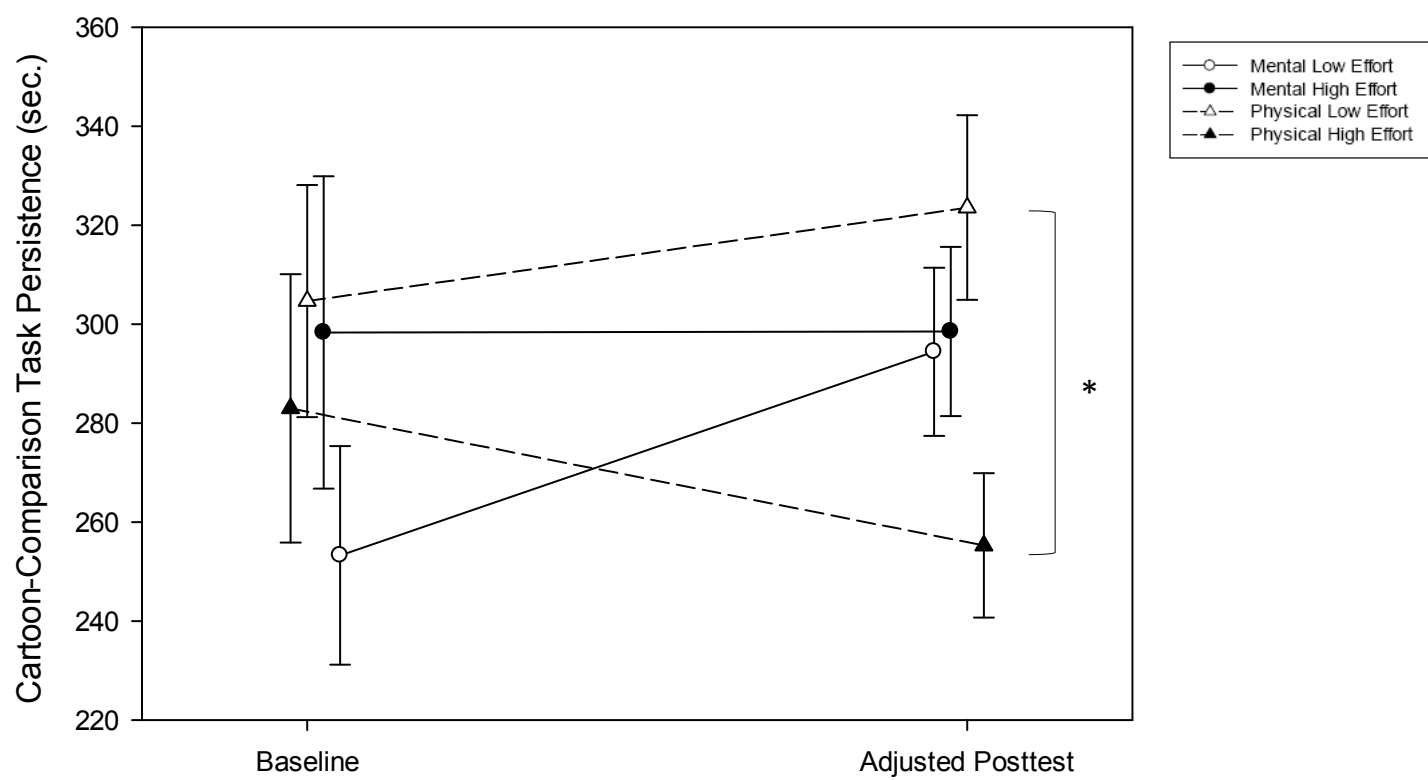

Figure 2. Baseline and adjusted posttest geometric means and standard errors for persistence by group

Note. Posttest adjusted for baseline persistence. * Low Physical Effort $>$ High Physical Effort $(p=.004)$.

Figure 3 presents unadjusted means and standard errors for points earned across groups from baseline to posttest. Between-group effect sizes on points earned were all near zero $(d=-0.12$ to 0.15$)$.

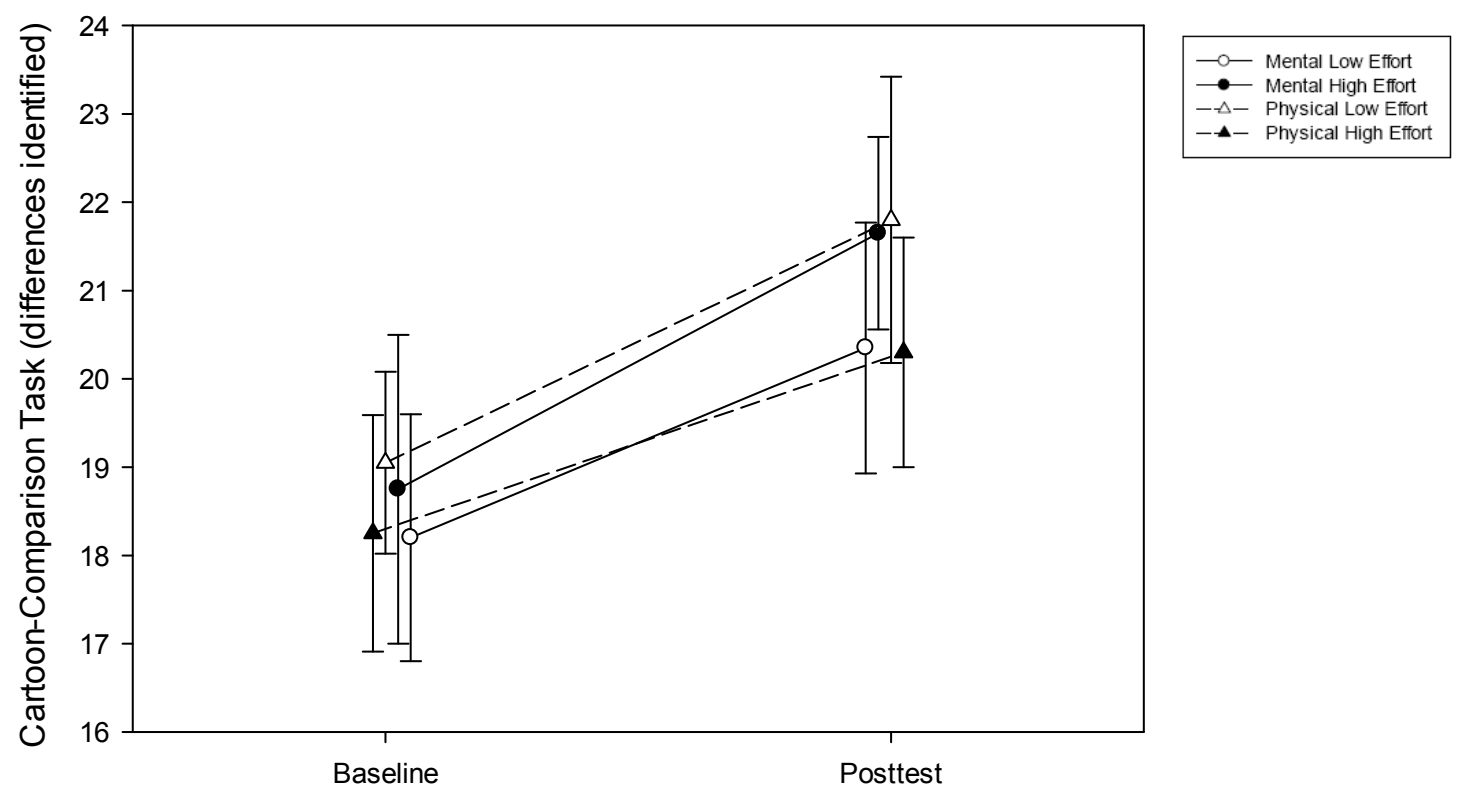

Figure 3. Points earned/differences identified on cartoon-comparison task at baseline and posttest (Mean $\pm \mathrm{SE})$

\section{Discussion}

Previous research has demonstrated that individuals reinforced for high effort during training demonstrate greater persistence at subsequent tasks than those rewarded for low effort. However, this has largely been evident only when the training and posttest were within the same domain (Eisenberger, 1992). Therefore, the extent to which reinforced physical activity generalizes effort to behaviors in the mental domain remains unknown. The current study adapted an established experimental design in Learned Industriousness research 
(Eisenberger \& Leonard, 1980, Experiment 1) by rewarding participants for completing a single bout of either light or vigorous intensity aerobic PA prior to discreetly timing them during a mental task. Findings reflect limitations of effort generalization between domains, as well as methodological considerations that arise when acute bout of aerobic physical activity is used as a training stimulus and only one brief period of contingent reward is used.

Learned Industriousness predicts that those rewarded for high effort should generalize greater effort to subsequent tasks than those rewarded for low effort regardless of domain. Yet the current study found a significant domain $x$ effort interaction reflecting a significant advantage of Low Physical Effort over High Physical Effort on persistence at a subsequent task. Similarly, Boyagian \& Nation (1981) failed to demonstrate generalization from physical effort training to persistence at a subsequent mental task in humans.

Eisenberger (1992) posited that the secondary reward value of effort is conditioned in relation to the stimulus context and generalizes according to the similarity between the effort-training situation and the transfer situation. If this is true, then the degree of primary stimulus generalization depends upon the number of stimulus elements shared between the effort-training and the transfer situation. Therefore, a possible explanation for the results of the current study and those of Boyagian and Nation (1981) is that the physical activity undertaken during training and the mental persistence task completed at posttest were too dissimilar to cue the associative learning expected to arise from effort training.

To reconcile the differences between these two studies and Eisenberger et al. (1979), which demonstrated transfer effects between custodial tasks and card-sorting, two methodological issues merit consideration. First, the current study and Boyagian and Nation (1981) utilized single exposures to lab-based physical effort tasks during training, whereas the effort training protocol in Eisenberger et al. (1979) utilized an unspecified variety of real-world custodial tasks over a period of weeks. This distinction is important because increasing the variety of tasks in which high performance is reinforced should condition the secondary reward value of effort to more stimulus elements and thereby produce greater transfer of high effort (Eisenberger, 1992).

Second, it could be argued that relative to Eisenberger et al. (1979), which utilized verbal praise from hospital staff subjects knew personally, the current study and Boyagian and Nation (1981) provided weaker rewards for effort (i.e., points on a scoreboard, verbal praise from research staff [the current study] and termination of an aversive beep [Boyagian \& Nation, 1981], respectively). This raises the possibility that the reward was not sufficiently desirable to allow for generalizable effects on effort. However, the current study utilized an equivalent reinforcement protocol to Eisenberger and Leonard (1981), which demonstrated generalization of effort across tasks reinforcing with points on a board and an audible beep. Moreover, Boyagian and Nation (1981) did demonstrate generalization of effort to an unrelated physical extinction task.

The current study added aerobic physical activity as a distinct component to the Learned Industriousness literature. The aim of this addition was to shed light on the potential of physical activity to improve persistence in other domains. However, despite adaptation of an established research protocol (Eisenberger \& Leonard, 1981), the lower persistence of the High Physical Effort group and the greater persistence of the Low Physical Effort group relative to the other groups suggest that findings were influenced by residual psychological and/or physiological effects of acute bouts of physical activity. For example, Tomporowski and Elliot (1986) suggested that physical activity may initially enhance cognitive performance by directly affecting the central nervous system; however as exercise intensity or duration increases cognitive benefits may be cancelled by debilitating effects of fatigue. Findings in the current study are consistent with that contention. Muraven, Tice and Baumeister (1998) demonstrated decrements in self-regulatory capacity following tasks requiring its exertion. Here it is possible that the vigorous intensity physical activity bout exhausted self-regulatory reserves beyond the other effort training modalities. Moreover, leading theoretical models of physical activity and affect suggest that affective responses to physical activity vary by individual characteristics, exercise intensity, and whether that intensity is self-selected or not (Ekkekakis, Parfitt, \& Petruzzello, 2011). In the current study's High Physical Effort group, physically inactive individuals were instructed to exercise at a moderate-vigorous intensity that they did not select. Consequently, the vigorous intensity physical activity bout may have led to feelings of lower activation and valence while the low-intensity physical activity bout may have led to higher activation and valence. Finally, Gailliot and Baumeister (2007) demonstrated that persistence is undermined by blood glucose depletion and restored with glucose feeding. Though this study did not measure blood glucose, it is unlikely that blood glucose depletion was the cause for the poorer persistence of the High Physical Effort group, since short bouts of moderate-vigorous physical activity rely disproportionately on muscle glycogen and triglycerides and plasma free fatty acids for energy (Coyle, 1995). 
The High Mental Effort and Low Mental Effort groups each demonstrated small improvements in persistence and points earned but between-group effect sizes were near zero. The null finding between mental effort groups makes this study the second to not replicate the persistence finding of Eisenberger and Leonard (1980), Experiment 1. Hickman, Stromme and Lippman (1998) randomized participants to high or low mental effort training or control. On a subsequent mental task, participants trained for high effort passed on fewer mazes than those trained for low effort and controls, but did not persist longer. The current study offered substantial methodological advantages over Hickman (1998) (e.g., a measure of baseline persistence, description of reinforcement protocol, and a larger sample size), but it nevertheless failed to reject the null hypothesis with regard to persistence among mental effort groups. It is possible the null result here was due to a smaller sample size than Eisenberger and Leonard (1980), Experiment 1, a limitation shared by Hickman (1998), thus, suggesting that a larger sample may have revealed a difference between mental effort groups. However, the utilization of a baseline measure of persistence in the current experiment is an advantage over those studies and between-group effect sizes for mental effort groups were near zero, suggesting that there was no advantage of rewarding higher effort for a single sitting in the current study, independent of sample size or domain. Furthermore, it was hypothesized that there would be a main effect on domain such that participants rewarded for mental effort would perform better on a post-training mental task than those rewarded for physical effort . This pattern was not evident, as small non-significant effects favored Low Physical Effort over Low Mental Effort and High Mental Effort over High Physical Effort. In each case, any potential advantage of task specificity appears to have been overridden by fatigue due to acute exercise bouts.

The current study represents a first step in the investigation of an established psychological theory, Learned Industriousness, in the context of physical activity, where it carries potentially meaningful implications. An experimental design previously utilized in Learned Industriousness research (Eisenberger \& Leonard, 1981, Experiment 1) was modified to incorporate rewards for effort on aerobic physical activity, and resulted in significant differences between groups on the primary outcome measure, persistence on a mental task. There were significant differences between groups during effort training on duration, points earned, heart rate, and rating of perceived exertion score in the expected directions suggesting that training intensity was manipulated as intended.

Future research on Learned Industriousness in the physical activity domain must adapt designs to control for residual effects of acute bouts of physical activity (i.e., fatigue). A more effective test of Learned Industriousness in the physical activity context will allow for recovery from fatigue-related effects deriving from vigorous aerobic physical activity. Therefore, future studies should consider extending the rest period to permit recovery from acute exercise or implementing effort training over days or weeks with posttest measures on the day after the final effort training session. This latter adaptation would also be advantageous because it would provide opportunities for the reinforcement of effort across a variety of physical activities, which is important because the Theory of Learned Industriousness states that stimulus variety enhances effort generalization (Eisenberger, 1992). Indeed, the most robust findings from the Learned Industriousness literature have come from studies in which individuals were rewarded for high effort over time (e.g., Eisenberger, et al., 1984; Eisenberger et al., 1979).

\section{Conclusion}

This study tested the hypothesis that reinforced high effort in the physical activity domain generalizes high effort to mental tasks using an adaptation of a previous experiment (Eisenberger \& Leonard, 1980, Experiment 1), which demonstrated this effect when varying intensities of mental effort were reinforced and subsequent mental persistence was measured. Results in physical effort groups were contrary to hypotheses as the training task may have been too dissimilar to the mental persistence outcome, and residual affective or physiologic consequences of vigorous physical activity may have inhibited effort generalization. The absence of differences between mental effort groups represents the second non-replication of the initial findings of Eisenberger and Leonard (1980, Experiment 1), likely reflecting the limited utility of rewarding effort in only a single sitting. Future experiments utilizing a multi-day or multi-week protocol would provide additional insight into whether effort learned in the physical activity domain can generalize to mental activities.

\section{Acknowledgements}

Funding for the current study was provided by the University of Illinois at Chicago, Department of Kinesiology and Nutrition. Special thanks are extended to Octavio Vega, Jacqueline Gomez, Jason Mikulich, Sonia Esperanza-Orozco, Roma Shah, Jeanette Agustin, Nicole Orseno, and the UIC Department of Psychology for their assistance with recruitment and data collection. 


\section{References}

Bandura, A. (1997). Self-Efficacy: The Exercise of Control. New York: Freeman.

Barkley, R. A. (1997). Behavioral inhibition, sustained attention, and executive functions: Constructing a unifying theory of ADHD. Psychological Bulletin, 121(1), 65-94. http://dx.doi.org/10.1037/0033-2909.121.1.65

Barkley, R. A. (1997a). ADHD and the Nature of Self-Control. New York: Guilford.

Bland, J. M., \& Altmant, D. G. (1996). Transformations, means, and confidence intervals. British Medical Journal, 312(7038), 1079. http://dx.doi.org/10.1136/bmj.312.7038.1079

Bonnet, M. H. (1980). Sleep, performance and mood after the energy-expenditure equivalent of 40 hours of sleep deprivation. Psychophysiology, 17(1), 56-63. http://dx.doi.org/10.1111/j.1469-8986.1980.tb02460.x

Boyagian, L. G. N. (1981). The effects of force training and reinforcement schedules on human performance. American Journal of Psychology, 94, 619-632. http://dx.doi.org/10.2307/1422423

Byron, K., \& Khazanchi, S. (2012). Rewards and creative performance: A meta-analytic test of theoretically derived hypotheses. Psychological Bulletin, 138(4), 809. http://dx.doi.org/10.1037/a0027652

Cameron, J., Pierce, W. D., \& So, S. (2004). Rewards, task difficulty, and intrinsic motivation: A test of learned industriousness theory. The Alberta Journal of Educational Research, 50(3), 317-320.

Carter, E. C., \& McCullough, M. E. (2013). After a pair of self-control-intensive tasks, sucrose swishing improves subsequent working memory performance. BMC Psychology, 1(1), 22. http://dx.doi.org/10.1186/2050-7283-1-22

Coe, D. P., Pivarnik, J. M., Womack, C. J., Reeves, M. J., \& Malina, R. M. (2006). Effect of physical education and activity levels on academic achievement in children. Med Sci Sports Exerc, 38(8), 1515-1519. http://dx.doi.org/10.1249/01.mss.0000227537.13175.1b

Cohen, J. (1988). Statistical Power for the Behavioral Sciences. Hillsdale, NJ: Lawrence Erlbaum Associates.

Conover, W. J., \& Iman, R. L. (1982). Analysis of covariance using the rank transformation. Biometrics, 715-724. http://dx.doi.org/10.2307/2530051

Costa, P. T., \& McCrae, R. R. (1989). The NEO-PI/NEO-FFI manual supplement. Odessa, FL: Psychological Assessment Resources.

Costa, P. T., \& Mccrae, R. R. (1992). The 5-Factor Model of Personality and Its Relevance to Personality-Disorders. Journal of Personality Disorders, 6(4), 343-359. http://dx.doi.org/10.1521/pedi.1992.6.4.343

Coyle, E. F. (1995). Substrate utilization during exercise in active people. The American Journal of Clinical Nutrition, 61(4), 968S-979S.

Duckworth, A. L., \& Seligman, M. E. (2005). Self-discipline outdoes IQ in predicting academic performance of adolescents. Psychological Science, 16(12), 939-944. http://dx.doi.org/10.1111/j.1467-9280.2005.01641.x

Eisenberger, R. (1992). Learned industriousness. Psychogical Review, 99(2), $248-267$. http://dx.doi.org/10.1037/0033-295X.99.2.248

Eisenberger, R., \& Aselage, J. (2008). Incremental effects of reward on experienced performance pressure: Positive outcomes for intrinsic interest and creativity. Journal of Organizational Behavior, 30(1), 95-117. http://dx.doi.org/10.1002/job.543

Eisenberger, R., Heerdt, W. A., Hamdi, M., Zimet, S., \& Bruckmeir, G. (1979). Transfer of persistence across behaviors. Journal of Experimental Psychology, 5(5), 522-530.

Eisenberger, R., \& Leonard, J. M. (1980). Effects of conceptual task difficulty on generalized persistence. America Journal of Psychology, 93(2), 285-298. http://dx.doi.org/10.2307/1422233

Eisenberger, R., Mitchell, M., McDermitt, M., \& Masterson, F. A. (1984). Accuracy versus speed in the generalized effort of learning-disabled children. Journal of the Experimental Analysis of Behavior, 42(1), 19-36. http://dx.doi.org/10.1901/jeab.1984.42-19

Ekkekakis, P., Parfitt, G., \& Petruzzello, S. J. (2011). The Pleasure and Displeasure People Feel When they Exercise at Different Intensities Decennial Update and Progress towards a Tripartite Rationale for Exercise 
$\begin{array}{llll}\text { Intensity } \quad \text { Prescription. } & \text { Sports }\end{array}$ http://dx.doi.org/10.2165/11590680-000000000-00000

Fox, S. M., Naughton, J. P., \& Haskell, W. L. (1971). Physical activity and the prevention of coronary heart disease. Annals of Clinical Research, 3(6), 404-432.

Gailliot, M. T., Baumeister, R. F., DeWall, C. N., Maner, J. K., Plant, E. A., Tice, D. M., Brewer, L. E., \& Schmeichel, B. J. (2007). Self-control relies on glucose as a limited energy source: Willpower is more than a metaphor. Journal of Personality and Social Psychology, 92, 325-336. http://dx.doi.org/10.1037/0022-3514.92.2.325

Gottfredson, M. R. H., T. (1990). A general theory of crime. Stanford, CA: Stanford University Press.

Hickman, K. L., Stromme, C., \& Lippman, L. G. (1998). Learned Industriousness: Replication in Principle. The Journal of General Psychology, 125(3), 213-217. http://dx.doi.org/10.1080/00221309809595545

Hollar, D., Messiah, S. E., Lopez-Mitnik, G., Hollar, T. L., Almon, M., \& Agatston, A. S. (2010). Effect of a Two-Year Obesity Prevention Intervention on Percentile Changes in Body Mass Index and Academic Performance in Low-Income Elementary School Children. American Journal of Public Health, 100(4), 646-653. http://dx.doi.org/10.2105/AJPH.2009.165746

James, W. (1890). Psychology. New York: Holt.

Jarrett, O. S., Maxwell, D. M., Dickerson, C., Hoge, P., Davies, G., \& Yetley, A. (1998). Impact of recess on classroom behavior: Group effects and individual differences. Journal of Educational Research, 92(2), 121-126. http://dx.doi.org/10.1080/00220679809597584

Kaufman, H., \& Schroeter, B. (1982a). Hocus-Focus 2: New American Library.

Kaufman, H., \& Schroeter, B. (1982b). Hocus-Focus 3: New American Library.

McAuley, E., Blissmer, B., Katula, J., \& Duncan, T. E. (2000). Exercise environment, self-efficacy, and affective responses to acute exercise in older adults. Psychology \& Health, 15(3), 341-355. http://dx.doi.org/10.1080/08870440008401997

McDougall, W. (1908). An introduction to social psychology. London: Methuen. http://dx.doi.org/10.1037/12261-000

Mischel, W., Shoda, Y., \& Peake, P. K. (1988). The nature of adolescent competencies predicted by preschool delay of gratification. Journal of Personal and Social Psychology, 54(4), 687-696. http://dx.doi.org/10.1037/0022-3514.54.4.687

Muraven, M., Tice, D. M., \& Baumeister, R. F. (1998). Self-control as limited resource: Regulatory depletion patterns. Journal of Personal and Social Psychology, 74(3), 774-789. http://dx.doi.org/10.1037/0022-3514.74.3.774

Nation, J. R., Cooney, J. B., \& Gartrell, K. E. (1979). Durability and generalizability of persistence training. Journal of Abnormal Psychology, 88(2), 121-136. http://dx.doi.org/10.1037/0021-843X.88.2.121

Nelson, M. C., \& Gordon-Larsen, P. (2006). Physical activity and sedentary behavior patterns are associated with selected adolescent health risk behaviors. Pediatrics, 117(4), 1281-1290. http://dx.doi.org/10.1542/peds.2005-1692

Oaten, M., \& Cheng, K. (2006). Longitudinal gains in self-regulation from regular physical exercise. British Journal of Health Psychology, 11, 717-733. http://dx.doi.org/10.1348/135910706X96481

Robins, R. W., Fraley, R. C., Roberts, B. W., \& Trzesniewski, K. H. (2001). A longitudinal study of personality change in young adulthood. Journal of Personality, 69(4), 617-640. http://dx.doi.org/10.1111/1467-6494.694157

Shoda, Y., Mischel, W., \& Peake, P. K. (1990). Predicting adolescent cognitive and self-regulatory competencies from preschool delay of gratification: Identifying diagnostic conditions. Developmental Psychology, 26, 978-986. http://dx.doi.org/10.1037/0012-1649.26.6.978

Thayer, R. E. (2001). Calm energy: How people regulate mood with food and exercise. New York: Oxford University Press.

Tomporowski, P. D., \& Ellis, N. R. (1986). Effects of exercise on cognitive processes: A review. Psychological Bulletin, 99(3), 338-345. http://dx.doi.org/10.1037/0033-2909.99.3.338 
Tresselt, M. E., \& Mayzner, M. S. (1966). Normative solution times for a sample of 134 solution words and 378 associated anagrams. Goleta, Calif.: Psychonomic Press.

Ventura, M., \& Shute, V. (2013). The validity of a game-based assessment of persistence. Computers in Human Behavior, 29(6), 2568-2572. http://dx.doi.org/10.1016/j.chb.2013.06.033

Ventura, M., Shute, V., \& Zhao, W. (2013). The relationship between video game use and a performance-based $\begin{array}{lllll}\text { measure of persistence. Computers \& } & \text { Education, } & 60(1), & 52-58 .\end{array}$ http://dx.doi.org/10.1016/j.compedu.2012.07.003

Wolfe, R. N., \& Johnson, S. D. (1995). Personality as a predictor of college performance. Educational and Psychological Measurement, 55(2), 177-185. http://dx.doi.org/10.1177/0013164495055002002

\section{Appendix A}

\section{Task Instructions}

At baseline, participants were presented with six cards and given the following instructions:

Here's a pile of cards. On each card there are two pictures. The pictures differ in several ways. Take a moment to examine the top card. Can you identify some differences? (Participants given 1-minute to identify some differences on practice card at top of pile.) When I say "go ahead", I want you to study each card and tell me as many differences as you can find between the two pictures. When you can't find any more differences on a card, turn to the next card. Please turn the cards in this fashion because they're ordered (demonstration using practice card). I will accumulate points for you on the scoreboard. I'll give you one point for each difference you discover between the two pictures on each card. Try to get as many points as you can for each card. (Name) will record your score and heart rate. I'll give you one point to start you off (experimenter registers 1-point on scoreboard). Are these instructions clear?

During effort training, participants in the Low Mental Effort and High Mental Effort groups received the following instructions:

Here's a pile of cards. Each card has some letters on it that can be unscrambled to form a word. When I say "go ahead", I want you to study the card. When you discover the word, tell me what it is. If you can't discover the word on a card, go on to the next card. Please turn the cards in this manner because they're ordered (demonstration on blank card). I will accumulate points for you on this scoreboard. Each time you discover a word, I'll give you a point. Try to get as many points as possible. (Name) will record your score and heart rate. I'll give you one point to start you off (experimenter registers 1-point on scoreboard). Are these instructions clear?

During effort training, Low Physical Effort and High Physical Effort participants were given the following instructions:

This is an electrical treadmill. When I start the treadmill I want you to walk, jog, or run at a pace that feels ____ to you (light-moderate for Low Physical Effort, moderate-vigorous for High Physical Effort). The heart rate monitor you have around your trunk will send a signal to the watch in (Name)'s hand informing him/her of your heart rate. (Name) will update us on the status of your heart rate after each minute. You will earn a point for each minute that you successfully keep your heart rate between _ and _\% of your maximum heart rate (45 and 60\% for Low Physical Effort, and $75-90 \%$ for High Physical Effort), or in your case ___ _ beats per minute. I will accumulate points for you on this scoreboard. Try to accumulate as many points as possible. You are free to adjust the speed of the treadmill in order to keep your heart rate within the desired range. So long as you feel safe, please stay on the treadmill for the full 10 minutes even if this means bringing your speed down to a very slow walk. If at any point you feel unsafe please do not hesitate to push the stop button and step off of the treadmill.

During the 5th and 9th minutes, I will present you with this scale (Borg RPE Scale), which is meant for you to report how hard you found the task. The top of the scale, number 6, means "no exertion at all" (experimenter points to top of scale) and the bottom of the scale, 20, means "maximal exertion" (experimenter points to bottom of scale). When I ask, simply point to the number on the scale that 
represents the level of exertion you felt during the task. For example, how hard do you feel you are working right now (participant should point to 6)? Okay, I will give you one point to start you off (experimenter registers 1-point on scoreboard). Are these instructions clear?

\section{Copyrights}

Copyright for this article is retained by the author(s), with first publication rights granted to the journal.

This is an open-access article distributed under the terms and conditions of the Creative Commons Attribution license (http://creativecommons.org/licenses/by/3.0/). 